notification is considered protective against fear of violence and social stigma.

Conclusions Meta-ethnography enabled the synthesis of qualitative literature to derive new interpretations and comparative explanations from these data. Our synthesis reveals that PN should be voluntary and confidential and a choice of patient and provider referral methods should be offered. PN programmes should support patients in coping with feelings of shame and guilt to assist notification and facilitate timely testing and treatment of sexual partners. Further research on the sexual health outcomes of $\mathrm{PN}$, its effect on partnerships and how best to reduce the fear of stigma and discrimination associated with STI and PN is needed.

\section{5-S3.06 TESTING FOR STIS IN POPULATION-BASED SEXUAL HEALTH SURVEYS WITHOUT RETURNING RESULTS: DEVELOPMENT OF AN ETHICAL AND PRAGMATIC APPROACH}

doi:10.1136/sextrans-2011-050109.168

${ }^{1} \mathrm{~N}$ Field, ${ }^{1} \mathrm{C}$ Tanton, ${ }^{1} \mathrm{C}$ Mercer, ${ }^{2} \mathrm{~S}$ Nicholson, ${ }^{2} \mathrm{~S}$ Sjordin, ${ }^{3} \mathrm{~K}$ Soldan, ${ }^{3} \mathrm{~S}$ Beddows, ${ }^{3} \mathrm{C}$ Ison, ${ }^{1}$ A Johnson, ${ }^{1} \mathrm{P}$ Sonnenberg. ${ }^{1}$ University College London, London, UK; ${ }^{2}$ National Centre for Social Research, London, UK; ${ }^{3}$ Health Protection Agency, London, UK

Background Sexual health surveys are enhanced by biological measures, such as non-invasive testing for STIs. The Third British National Survey of Sexual Attitudes and Lifestyles (Natsal 3) will estimate the population prevalence of five STIs ( $C$ trachomatis, $N$ gonorrhoeae, type-specific Human Papilloma Virus, HIV and $M$ genitalium) in a probability sample aged $16-44 \mathrm{y}$. This raises complex ethical issues, including return of results, testing in under-18s, appropriate consent and confidentiality. We describe the development of an ethically sound and acceptable approach to urine testing for STIs, without returning results, in Natsal 3.

Methods The Natsal team considered the following: (1) more appropriate settings to obtain free STI testing and advice are now widely available, (2) survey conditions and sample type limit accuracy and timeliness, (3) for some STIs, the clinical and public health implications are unknown, (4) a uniform approach is easier to explain and be understood, (5) non-return of results is more cost efficient, enabling wider STI testing, (6) practical difficulties in returning results, (7) non-clinical interviewers. This rationale was subjected to peer-review as part of the funding process, stakeholders were engaged, and it was then submitted to a research ethics committee (REC). Piloting was performed in the general population to test acceptability and feasibility. Findings The agreed approach involved written consent for voluntary anonymised testing with the knowledge that results are not returned and the option of sample storage for future research. Confidentiality was assured by keeping STI data and personal identifiers at separate institutions, and a detailed protocol was designed to ensure that STI data were irreversibly unlinked after 3 months. In piloting, $61 \%(68 / 111)$ of participants agreed to provide a sample and $93 \%$ $(63 / 68)$ agreed to sample storage for future research. All participants were given information about accessing clinical tests and STI advice.

Conclusions The testing of STIs and the decision not to return results was accepted by a REC, in the context of a stakeholder consensus, and was acceptable to participants in developmental pilot work. Our experiences, both in developing the approach, and in the mechanisms to achieve this, may inform the ethical considerations of researchers, RECs and funders in study design and ethical approaches to population-based biological sampling.

\section{Health services and policy oral session 4-Screening 05-S4.01 REGISTER-BASED CHLAMYDIA SCREENING: DOES IT
MAKE A DIFFERENCE IF THE INVITATION IS SENT BY GP
OR BY MUNICIPAL HEALTH SERVICE?}

doi:10.1136/sextrans-2011-050109.169

${ }^{1} \mathrm{~J}$ E A M van Bergen, ${ }^{2} \mathrm{R}$ H Koekenbier, ${ }^{2} \mathrm{~J} S \mathrm{~S}$ Fennema, ${ }^{3} \mathrm{E}$ E H G Brouwers, ${ }^{4} \mathrm{H}$ M Götz, ${ }^{3} \mathrm{C}$ J P A Hoebe, ${ }^{5} \mathrm{E}$ L M Op de Coul, ${ }^{1} \mathrm{~L}$ L Pars, ${ }^{6}$ S M van Ravensteijn, I V F van den Broek. ' Soa Aids Nederland, Amsterdam, Netherlands; ${ }^{2}$ Public Health Service, Amsterdam, Netherlands; ${ }^{3}$ Public Health Service, South-Limburg, Netherlands; ${ }^{4}$ Public Health Service, Rotterdam Rijnmond, Netherlands; ${ }^{5}$ Centre for Infectious Disease Control RIVM, Netherlands; ${ }^{6}$ Public Health Service, Rotterdam-Rijnmond, Netherlands

Background In cervical cancer screening, uptake of screening is approximately $10 \%$ higher if a woman is invited by her General Practitioner (GP) compared to invitation by Municipal Public Health Service (PHS). A large register-based selective Chlamydia Screening Programme is implemented in Amsterdam (Netherlands) since 2008 by the PHS. We wanted to evaluate whether response rates in Chlamydia screening where higher if invitational letters are sent by a persons' GP and not by PHS.

Methods Based on the municipal registers, annually all 140000 persons in Amsterdam aged 16-29 yr are invited with a postal letter to participate. Further procedures are internet-based: requesting testpackages, obtaining results, even partner-referral, can be realised via the internet and the unique identifier provided in the personal letter. In one part of the city, the Southeast inner-city area, GP-registers of six health centre are matched with the list of invitees extracted from the municipal register. In the invitational letter these matched eligible's are personally addressed by their GP and not by the physician of the PHS. Participation rates from invitees of GP and $\mathrm{PHS}$ in the same geographical town area are compared in three screenings-rounds (r 3 not yet fully completed).

Results In these four town area's in each screening round annually 13500 persons are invited, of which $35 \%$ by the GP and $65 \%$ by PHS. In screenings-round 1, the response to invitations by the GP was slightly higher than the response to invitations sent by the PHS in 3 out of 4 neighbourhoods where this was piloted (overall GPs: 16.2\% [range: 15.3\%-29.1\%]; overall PHS: $14.9 \%$ [14.4\%-21.6\%], $p=0.023)$. In round 2 and 3 the difference was not significant (GP vs MHS: r2 $10.3 \%-10.2 \%$; r3 9.5\% vs 9.4\%). (Abstract O5-S4.01 figure 1).

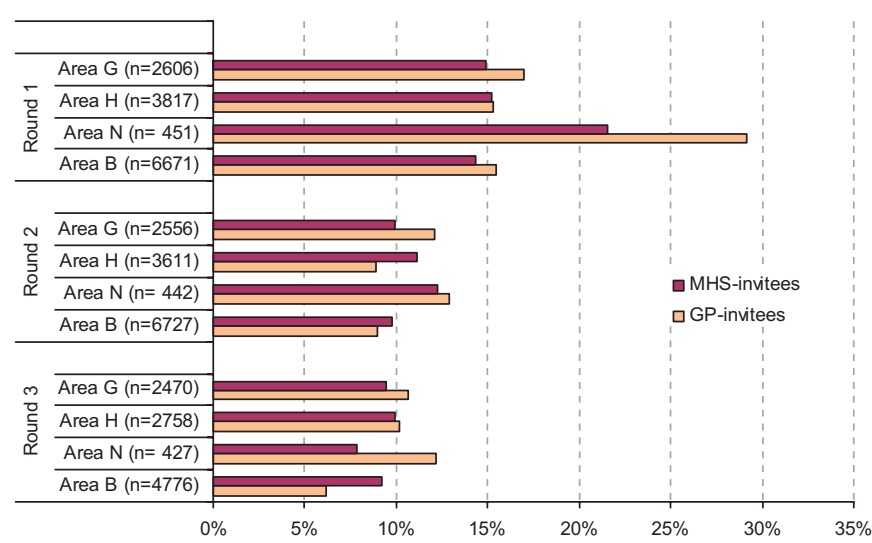

Abstract 05-S4.01 Figure 1 Participation rate comparing response among GP- and MHS-invitees in 4 town-areas of Amsterdam Southeast.

Conclusions In the first screening-round uptake was slightly higher among GP-invited, but this effect was lost in subsequent screeningrounds. Inviting universally via the PHS is logistically easier and yields the same response rate. 